\title{
Molecular identification and assessment of genetic diversity of fluorescent pseudomonads based on different polymerase chain reaction (PCR) methods
}

\author{
Inès Mehri ${ }^{1 \star}$, Yousra Turki ${ }^{1}$, Imen Daly ${ }^{1}$, Asma Ben Rjab ${ }^{1}$, Abdennaceur Hassen ${ }^{1}$ and Maher \\ Gtari $^{2}$ \\ ${ }^{1}$ Laboratoire Traitement et Recyclage des Eaux, Centre de Recherche et des Technologies des Eaux, Borj-Cédria, \\ Tunisia. \\ ${ }^{2}$ Laboratoire Microorganismes et Biomolécules actives Faculté des Sciences de Tunis, Campus Universitaire, 2092, \\ Tunis, Tunisia.
}

Accepted 15 February, 2013

\begin{abstract}
A total of 66 fluorescent pseudomonads strains isolated from diverse Tunisian environmental biotypes (wastewater, compost, wastewater treatment plant, etc.) were analysed by two polymerase chain reaction (PCR)-based methods, 16S-23S intergenic spacer regions (ITS)-PCR and repetitive extragenic palindromic (BOX)-PCR. These typing techniques were evaluated to assess their usefulness as tool to study the Pseudomonas diversity within this complex group. Genetic analysis using ITS- and BOX-PCR generated respectively 12 and 45 distinct profiles. Phylogenetic relationships within fluorescent pseudomonads were examined by analyzing partial 16S rRNA and rpoB genes sequences. The phylogenetic resolution of the rpoB tree was higher than that of the 16S rRNA tree. Moreover, the sequencing of the rpoB gene has recognized 13 different species and sub-species, while the 16 rRNA gene sequencing differentiated only 9 species.
\end{abstract}

Key words: Fluorescent pseudomonads, 16S-23S intergenic spacer regions (ITS)-PCR, repetitive extragenic palindromic (BOX)-PCR, 16S rRNA, rpoB.

\section{INTRODUCTION}

The genus Pseudomonas, firstly described by Migula in 1894 , is characterized as straight or slightly bent Gram negative rods with one or more polar flagellae, not forming spores (Fuchs et al., 2001). Its metabolism is chemoorganotrophic and strictly aerobic with a respiratory type in which oxygen is used (Fuchs et al., 2001).

Pseudomonas "sensu stricto" group I is the largest of the groups, and includes both fluorescent and non fluorescent ones. The most important fluorescent species are Pseudomonas aeruginosa, Pseudomonas fluorescens, Pseudomonas putida and plant pathogenic species (Pseudomonas syringae) (Scarpellini et al., 2004). Several species of rRNA group I pseudomonads have the ability to produce and excrete, under condition of iron limitation, soluble yellow green pigments that fluorescence under UV light (Bultreys et al., 2003), named pyoverdines (PVDs) or pseudobactins, which act as siderophores for these bacteria (Meyer, 2000). These molecules are thought to be associated with biocontrol of fungal pathogens in the biosphere (Fuchs et al., 2001).

The abundance of literature on genus Pseudomonas, is due to their elevated metabolic versatility capable of utilizing a wide range of simple and complex organic compounds and holding an important position in biosphere ecology (Scarpellini et al., 2004). Consequently, they are isolated from a variety of natural sources including soil, plants and mineral waters and 
from clinical specimens and they are characterized by a high level of metabolic diversity (Moore et al., 1996). Often, they are able to survive and multiply in poor nutrient conditions (Moreira et al., 1994).

Fluorescent pseudomonads have been considered as an important bioinoculants due to their innate potential to produce plant growth promoting hormones (Latour et al., 2003) and antimicrobial secondary metabolites (Costa et al., 2006; Dong and Zhang, 2005).

Considering the multiple applications of fluorescent pseudomonads, it is essential to study their diversity, which will be useful in designing strategies to use these strains as bioinoculants.

The characterization of Pseudomonas genus is faced with difficulties based on their genetic heterogeneity. Recently, the development of molecular techniques has yielded innovative alternative tools for demonstrating the mechanisms underlying biocontrol properties (Massart and Jijakli, 2007) and understanding the role of these bacteria in bioremediation, plant spoilage and pathogenicity (Ravi Charan et al., 2011).

Microbial DNA fingerprinting has been reported extensively using polymerase chain reaction (PCR)based techniques such as ITS- and BOX-PCR. The 16S23S polymorphism has been used to differentiate among Gram negative bacteria at the species as well as the genus level and to assess the phylogenetic closeness among bacteria (Ouzari et al., 2008; Wang and Jayarao, 2001). Other methods appeared interesting for the delineation of species, subspecies (Louws et al., 1998) or pathovars (Louws et al., 1994) now available. Box-PCR is the most commonly used technique due to its simplicity, efficiency and low cost (Brusetti et al., 2008). This is a particular version of repetitive extragenic palindromicPCR (rep-PCR) that uses the BOX-A1R primer (Versalovic et al., 1991). BOX-PCR, has revealed the possibility of delineating $P$. syringae genomospecies (Marques et al., 2000), as well as for typing Aeromonas spp. strains (Tacao et al., 2005).

On the other hand, the $16 \mathrm{~S}$ rRNA and rpoB gene sequences have been widely used to assess phylogenetic closeness among bacteria. In many cases, the 16S rRNA gene sequence is not sufficiently discriminative for taxonomic purposes (Adékambi et al., 2009). The high degree of conservation of $r$ rs gene led to a small number of informative sites in its sequence (Ait Tayeb et al., 2005) and it fails to reveal precise and statistically supported phylogeny at the species level (Hasegawa and Hashimoto, 1993). Consequently, several attempts have been made to identify other genes, which can be used to determine the relatedness between species, such as $g y r B, r p o D$, ampC, oprl, pilA, etc. for Pseudomonas genus (Bennasar et al., 2010). The rpoB gene, which codes for the RNA polymerase beta subunit, has been used as signature for bacterial identification for phylogenetic analysis (Ait Tayeb et al., 2005).

The aim of this investigation was to distinguish the different haplotypes of fluorescent Pseudomonas collection by combining two PCR-based molecular methods. In addition, we identified representatives of the abundant groups by $16 \mathrm{~S}$ rDNA and rpoB sequencing.

\section{MATERIALS AND METHODS}

\section{Bacterial strains}

Pseudomonas strains described in the present study were collected over a period of 18 months from environment and clinical origin. Environmental samples were transported to the laboratory in sterile stomacher bags, stored at $4^{\circ} \mathrm{C}$, and analyzed within $24 \mathrm{~h}$. Strains whose designations begin respectively with PsWw, PsWs and PsWt; were collected from water (waste water, sea water and thermal water); strains whose designations begin with PsS were collected from soil and strains whose designations begin respectively with $\mathrm{PSC}$ and $\mathrm{PSTp}$ were isolated from compost and waste water treatment plant. Clinical strains are designed as PSCL.

\section{Isolation and growth conditions}

Ten grams or $10 \mathrm{ml}$ of sample were suspended in $90 \mathrm{ml}$ sterile distilled water. Fifty microliters of an appropriate serial dilution was spread on King's B (KB) and King's A (KA) agar medium (Difco Laboratories), and one of each of the phenotypically different colonies developing a fluorescent halo after $48 \mathrm{~h}$ of incubation at $25^{\circ} \mathrm{C}$ was further purified by streaking it on the same medium (Munsch et al., 2000). Routine growth was in Luria-Bertani (LB) medium or King's B liquid medium (King et al., 1954).

For this study, putative Pseudomonas colonies were collected from representative plates and initially characterized by Gram staining and testing for oxidase and catalase activities. All single colonies that were confirmed as putative Pseudomonas spp. (Gram negative, oxidase positive and catalase positive) were used for further characterization as described below. Strains were preserved by mixing overnight LB culture with $50 \%$ glycerol $(1: 1$, vol $/ \mathrm{vol})$ and storage at $-80^{\circ} \mathrm{C}$.

The fluorescence was detected in the iron-poor liquid growth medium (Meyer et al., 2002), the casamino acid (CAA) medium, consisting of (per liter) $5 \mathrm{~g}$ of low-iron Bacto casamino acid (Difco), $1.54 \mathrm{~g}$ of $\mathrm{K}_{2} \mathrm{HPO}_{4} \cdot 3 \mathrm{H}_{2} \mathrm{O}$ and $0.25 \mathrm{~g}$ of $\mathrm{MgSO}_{4} \cdot 7 \mathrm{H}_{2} \mathrm{O}$.

\section{Genomic DNA extraction}

A single bacterial colony was inoculated into $5 \mathrm{ml}$ (LB) and grown for $16 \mathrm{~h}$ at $30^{\circ} \mathrm{C}$. Saturated culture was harvested with centrifugation for $3 \mathrm{~min}$ at $12,000 \mathrm{rpm}$. The cell pellet was resuspended and lysed in $200 \mu \mathrm{l}$ of lysis buffer (40 mM Tris-acetate $\mathrm{pH}$ 7.8, $20 \mathrm{mM}$ sodium-acetate, $1 \mathrm{mM}$ EDTA, 1\% SDS) by vigorous pipetting. To remove most proteins and cell debris, $66 \mu \mathrm{l}$ of $5 \mathrm{M}$ $\mathrm{NaCl}$ solution was added and mixed well, and then the viscous mixture was centrifuged at $12,000 \mathrm{rpm}$ for $10 \mathrm{~min}$. An equal volume of chloroform was added to the clear supernatant. Following centrifugation at 12,000 rpm for $3 \mathrm{~min}$, the extract supernatant was precipitated with $100 \% \mathrm{EtOH}$, washed twice with $70 \% \mathrm{EtOH}$, dried and redissolved in $50 \mu \mathrm{l}$ 1x TE buffer (Chen and Kuo, 1993).

\section{PCR amplification}

All the PCR (except BOX-PCR) were performed in a volume of 50 $\mu \mathrm{l}$ of bacterial genomic DNA solution, $10 \mu \mathrm{l}$ of $5 \times$ PCR buffer, 100 
$\mu \mathrm{M}$ of each dNTP, $1 \mathrm{mM}$ of $\mathrm{MgCl}_{2}, 0.25 \mu \mathrm{M}$ of each primer and $1 \mathrm{U}$ of Taq Polymerase.

Polymorphisms were sought in the 16S-23S spacer region of the rRNA genes by DNA amplification using the primers complimentary to the conserved regions of the $16 S$ and $23 S$ bacterial rRNA genes. DNA 16S-23S intergenic spacer (ITS) region amplification was performed using the primer set 16F945 and 23R458 (16F945 5'GGGCCCGCACAAGCGGTGG-3'; 23R458

5 CTTTCCCTCACGGTAC-3') (Lane et al., 1985) and the following thermal profile: 4 min at $94^{\circ} \mathrm{C} ; 40$ cycles consisting of $94^{\circ} \mathrm{C}$ for $30 \mathrm{~s}$, $45^{\circ} \mathrm{C}$ for $30 \mathrm{~s}, 72^{\circ} \mathrm{C}$ for $45 \mathrm{~s}$; final extension of $72^{\circ} \mathrm{C}$ for $7 \mathrm{~min}$.

BOX-PCR was performed as described by Brusetti et al. (2008). Mixtures contained $1 \times \mathrm{PCR}$ buffer, $2 \mathrm{mM} \mathrm{MgCl} 2,0.1 \mathrm{mM}$ dNTPs, $0.8 \mu \mathrm{M}$ of BOX-A1R primer, $5 \%$ of dimethylsulfoxide, $1.3 \mathrm{U}$ of Taq DNA polymerase and standardized $15 \mathrm{ng}$ of genomic DNA in a final volume of $30 \mu \mathrm{l}$. Reactions were denatured at $94^{\circ} \mathrm{C}$ for $5 \mathrm{~min}$, subjected to 35 cycles of $94^{\circ} \mathrm{C}$ for $1 \mathrm{~min}, 45^{\circ} \mathrm{C}$ for $1 \mathrm{~min}$ and $72^{\circ} \mathrm{C}$ for $2 \mathrm{~min}$ and a final extension at $72^{\circ} \mathrm{C}$ for $10 \mathrm{~min}$.

The discriminatory power of ITS- and BOX-PCR methods was calculated by application of Simpson numerical index of diversity (Hunter and Gaston, 1988).

DNA 16S region amplification was performed using selective primers to amplify a 969-bp fragment: forward primer Ps-for (20-mer [5'-GGTCTGAGAGGATGATCAGT-3']) and reverse primer Ps-rev (18-mer [5'-TTAGCTCCACCTCGCGGC-3']) (Widmer et al., 1998). The PCR program used was: 4 min at $94^{\circ} \mathrm{C} ; 30$ cycles consisting of $94^{\circ} \mathrm{C}$ for $30 \mathrm{~s}, 60^{\circ} \mathrm{C}$ for $30 \mathrm{~s}, 72^{\circ} \mathrm{C}$ for $45 \mathrm{~s}$; final extension of $72^{\circ} \mathrm{C}$ for $7 \mathrm{~min}$; and final cooling at $16^{\circ} \mathrm{C}$.

RpoB region amplification was carried out using selective primers to amplify a 1247-bp fragment: LAPS (5'TGGCCGAGAACCAGTTCCGCGT-3') and LAPS-27 (CGGCTTCGTCCAGCTTGTTCAG) (Ait Tayeb et al., 2005). The PCR program used was: $94^{\circ} \mathrm{C}$ for 4 min, 40 cycles of denaturation at $94^{\circ} \mathrm{C}$ for $30 \mathrm{~s}$, annealing at $52^{\circ} \mathrm{C}$ for $30 \mathrm{~s}, 72^{\circ} \mathrm{C}$ for $45 \mathrm{~s}$ and a final extension step at $72^{\circ} \mathrm{C}$ for $7 \mathrm{~min}$.

PCR amplifications were performed with a Biometra UNOII Thermal Cycler. $5 \mu \mathrm{l}$ of PCR products were analysed by electrophoresis at $100 \mathrm{~V}$ respectively on $(2,2,1.5$ and $1 \%)$ agarose gels for ITS-PCR, BOX-PCR, 16S rDNA and rpoB, containing 0.2 $\mu \mathrm{g}$ of ethidium bromide $\mathrm{ml}^{-1}$ in TAE buffer and photographed using UV light.

\section{Partial sequencing of the 16S-rDNA and rpoB}

PCR products obtained from representative BOX and ITS groups (34 bacterial isolates) were purified with a Promega PCR purification Kit, and sequenced using an Applied Biosystems sequencer. Sequences of the PCR products were aligned and corrected manually with Chromas Pro (version 1.34). The BLAST database of the National Center for Biotechnology Information (NCBI) was used to compare resolved sequences of the 34 isolates with 16S rDNA and rpoB sequences data deposited in GenBank. Alignment of sequences was performed using the Clustal method (ClustalX (1.81)). Analysis of the distance between sequences was made with aid of the phylogenetic analysis using the Fitch program within the Phylogeny Inference Package (PHYLIP).

The NCBI accession numbers for the 16S rRNA gene and rpoB gene sequences of 34 representative isolates determined in this present study are listed in Table 1.

\section{RESULTS}

A total of 66 pseudomonads isolates showing fluorescence under UV light, isolated over a period of 18 months from diverse origin, were spread on King's $B$ $(\mathrm{KB})$.

\section{ITS- and BOX-PCR fingerprinting}

Figure 1 represents the patterns obtained with ITS-PCR tested with studied Pseudomonas fluorescent population (only 32 representative strains are shown). This method applied to 66 isolates relies on polymorphisms within the 16S-23S intergenic spacer regions. A characteristic pattern is obtained that represents the size variations in the multiple rRNA operons. ITS technique showed reproducible patterns consisting of 1 to 4 bands ranging from 300 to $750 \mathrm{bp}$, but the majority of strains contained only one band. 12 different ITS profiles were produced, encompassing 21 (G1), 1 (G2), 1 (G3), 3 (G4), 1 (G5), 5 (G6), 16 (G7), 1 (G8), 14 (G9), 1 (G10), 1 (G11) and 1 (G12) isolates (Table 1). The discriminatory power of ITS technique was calculated to be 0.797 .

Figure 2 represents the pattern obtained with BOXPCR tested with studied Pseudomonas fluorescent population. To gain insight into the genetic diversity and structure of the isolates, BOX-A1R-based repetitive extragenic palindromic-PCR was applied to the 66 isolates. Visual inspection of the DNA fingerprints followed by analysis with Gel-Pro (version 3.1), demonstrated 2 to 12 reproducible bands varying from 100 to 2500 bp with different intensity. 45 distinct profiles were obtained enclosing seven major groups (I to VII) (Table 1). The discriminatory power of Box-PCR method was calculated to be 0.973 .

\section{S rDNA and rpoB sequence analysis and phylogenetic tree}

Figures 3 and 4 represent phylogenetic trees obtained with $16 \mathrm{~S}$ rRNA and rpoB sequences determined in this study for the same strains and the branches with bootstrap value above $70 \%$ are shown with spots at nodes.

On the basis of BLAST analysis of 16S rRNA gene (about $700 \mathrm{bp}$ ) similarity, species such as $P$. aeruginosa, Pseudomonas putida, Pseudomonas plecoglossicida, Pseudomonas monteilii, Pseudomonas mosselii, $P$. fluorescens, Pseudomonas thivervalensis, Pseudomonas otitidis and Pseudomonas guezennei were identified. A total of 19 isolates (PsS2, PsS71, PsS15, PsS28, PsS31, PsS46, PsS48, PsC54, PsS67, PsS75, PsS79, PsWs147, PsTp156, PsC10, PsTp139, PsWs173, PsS83, PsWs140 and PsWt146) belong to P. putida / P. plecoglossicida, one isolate (PsS11) belongs to $P$. monteilii, one isolate (PsTp171) belongs to P. mosselii, six isolates (PsS26, PsS29, PsS73, PsS93, PsS89 and PsWw128) belong to $P$. fluorescens / $P$. thivervalensis, five isolates (PsWw84, PsS150, PsS3, PsC132 and 
Table 1. Genetic diversity among pseudomonas strains.

\begin{tabular}{|c|c|c|c|c|c|c|c|}
\hline Isolates & $\begin{array}{l}16 \mathrm{~S} \\
\text { accession } \\
\text { number }\end{array}$ & rDNA & $\begin{array}{l}\text { Closest } 16 S \quad \text { rDNA } \\
\text { sequence with type strain }\end{array}$ & $\begin{array}{l}\text { rpoB } \\
\text { accession } \\
\text { number } \\
\end{array}$ & $\begin{array}{l}\text { Closest rpoB } \\
\text { sequence with type } \\
\text { strain }\end{array}$ & $\begin{array}{l}\text { ITS } \\
\text { profile }\end{array}$ & $\begin{array}{l}\text { BOX } \\
\text { profile }\end{array}$ \\
\hline PsS150 & HM627571 & & $P$. aeruginosa & JN590039 & $P$. aeruginosa & G1 & $\mathrm{III}_{2}$ \\
\hline PsWt157 & & & & & & G1 & $\mathrm{I}_{4}$ \\
\hline PsWw168 & & & & & & G1 & $\mathrm{III}_{7}$ \\
\hline PsWw175 & & & & & & G1 & $\mathrm{I}_{2}$ \\
\hline $\mathrm{PsC} 132$ & HM627574 & & $P$. aeruginosa & JN627242 & $P$. aeruginosa & G12 & $\mathrm{III}_{5}$ \\
\hline PsWt138 & & & & & & G1 & $\mathrm{I}_{3}$ \\
\hline PsCL10C & & & & & & G1 & $\mathrm{I}_{1}$ \\
\hline PsTp160 & & & & & & G1 & $\mathrm{II}_{2}$ \\
\hline PsC99 & & & & & & G1 & $\mathrm{II}_{1}$ \\
\hline PsTp179 & & & & & & G1 & $\mathrm{I}_{2}$ \\
\hline $\mathrm{PsC5}$ & & & & & & G1 & $\mathrm{I}_{4}$ \\
\hline PsWw174 & & & & & & G1 & $\mathrm{III}_{3}$ \\
\hline PsS2 & HM627611 & & $\begin{array}{l}\text { P. putida / } \\
\text { P. plecoglossicida }\end{array}$ & JN701899 & $\begin{array}{l}\text { P. putida / } \\
\text { P. syncynea }\end{array}$ & G11 & $\mathrm{III}_{1}$ \\
\hline PsS3 & HM627570 & & $P$. aeruginosa & JN620413 & $P$. aeruginosa & G1 & $\mathrm{I}_{5}$ \\
\hline PsCLHMC1 & & & & & & G1 & $\mathrm{III}_{2}$ \\
\hline PsWw127 & & & & & & G1 & $\mathrm{III}_{3}$ \\
\hline $\mathrm{PsC} 12$ & & & & & & G1 & $\mathrm{III}_{3}$ \\
\hline PsWw84 & HM627575 & & $P$. aeruginosa & JN590033 & $P$. aeruginosa & G1 & $\mathrm{III}_{6}$ \\
\hline PsWw121 & HM627577 & & $P$. aeruginosa & JN590036 & P. otitidis & G1 & $\mathrm{VI}_{2}$ \\
\hline PsTp142 & & & & & & G1 & $\mathrm{VI}_{2}$ \\
\hline PsS89 & HM627583 & & $\begin{array}{l}\text { P. fluorescens / } \\
P . \text { thivervalensis }\end{array}$ & JN590034 & P. moraviensis & G1 & $\mathrm{VII}_{5}$ \\
\hline PsWw9 & & & & & & G1 & $\mathrm{Vl}_{2}$ \\
\hline PsTp139 & HM627618 & & $\begin{array}{l}\text { P. putida / } \\
\text { P. plecoglossicida }\end{array}$ & JN660816 & Pseudomonas sp. & G2 & $\mathrm{IV}_{13}$ \\
\hline PsWs140 & HM627619 & & $\begin{array}{l}\text { P. putida / } \\
\text { P. plecoglossicida }\end{array}$ & JN590037 & P. monteilii & G3 & $\mathrm{IV}_{13}$ \\
\hline PsTp153 & & & & & & G4 & $\mathrm{IV}_{13}$ \\
\hline PsTp154 & & & & & & G4 & $\mathrm{IV}_{13}$ \\
\hline PsTp155 & & & & & & G4 & $\mathrm{IV}_{13}$ \\
\hline PsWw128 & HM627582 & & $\begin{array}{l}P . \quad \text { fluorescens } / \quad P . \\
\text { thivervalensis }\end{array}$ & KC161437 & P. vancouverensis & G5 & $\mathrm{VII}_{4}$ \\
\hline PsTp169 & & & & & & G6 & $\mathrm{IV}_{5}$ \\
\hline Isolates & $\begin{array}{l}16 S \\
\text { accession } \\
\text { number }\end{array}$ & rDNA & $\begin{array}{l}\text { Closest 16S rDNA } \\
\text { sequence with type strain }\end{array}$ & $\begin{array}{l}\text { rpoB } \\
\text { accession } \\
\text { number }\end{array}$ & $\begin{array}{l}\text { Closest rpoB } \\
\text { sequence with type } \\
\text { strain }\end{array}$ & $\begin{array}{l}\text { ITS } \\
\text { profile }\end{array}$ & $\begin{array}{l}\text { BOX } \\
\text { profile }\end{array}$ \\
\hline PsS11 & HM627617 & & P. monteilii & JN634561 & Pseudomonas sp. & G6 & $\mathrm{IV}_{12}$ \\
\hline PsTp172 & & & & & & G6 & $\mathrm{IV}_{5}$ \\
\hline PsS103 & & & & & & G6 & $\mathrm{IV}_{8}$ \\
\hline PsS71 & HM627580 & & $\begin{array}{l}\text { P. putidal } \\
\text { P. plecoglossicida }\end{array}$ & JN701900 & $\begin{array}{l}\text { P. putida / } \\
\text { P. syncynea }\end{array}$ & G7 & $\mathrm{IV}_{11}$ \\
\hline PsC10 & HM627622 & & $\begin{array}{l}\text { P. putida / } \\
\text { P. plecoglossicida }\end{array}$ & JN603371 & Pseudomonas sp. & G7 & $\mathrm{V}_{2}$ \\
\hline PsS73 & HM627594 & & $\begin{array}{l}\text { P. fluorescens / } \\
\text { P. thivervalensis }\end{array}$ & JN704638 & $\begin{array}{l}\text { P. aurantiaca / } \\
\text { P. chlororaphis }\end{array}$ & G7 & $\mathrm{VII}_{8}$ \\
\hline PsS26 & HM627593 & & $\begin{array}{l}\text { P. fluorescens / } \\
\text { P. thivervalensis }\end{array}$ & JN704637 & $\begin{array}{l}\text { P. aurantiaca / } \\
\text { P. chlororaphis }\end{array}$ & G7 & $\mathrm{VII}_{7}$ \\
\hline
\end{tabular}


Table 1. Contd.

\begin{tabular}{|c|c|c|c|c|c|c|}
\hline PsS29 & HM627585 & $\begin{array}{l}\text { P. fluorescens / } \\
P . \text { thivervalensis }\end{array}$ & JN660815 & $\begin{array}{l}\text { P. aurantiaca / } \\
\text { P. chlororaphis }\end{array}$ & G7 & $\mathrm{VII}_{6}$ \\
\hline PsS39 & & & & & G7 & $\mathrm{VII}_{6}$ \\
\hline PsS25 & & & & & G7 & $\mathrm{VII}_{6}$ \\
\hline PsS49 & & & & & G7 & $\mathrm{VII}_{6}$ \\
\hline PsS60 & & & & & G7 & $\mathrm{VII}_{6}$ \\
\hline PsS93 & HM627589 & $\begin{array}{l}\text { P. fluorescens / } \\
\text { P. thivervalensis }\end{array}$ & JN627241 & $\begin{array}{l}\text { P. aurantiaca / } \\
\text { P. chlororaphis }\end{array}$ & G7 & $\mathrm{VII}_{6}$ \\
\hline PsS90 & & & & & G7 & $\mathrm{VII}_{6}$ \\
\hline PsS91 & & & & & G7 & $\mathrm{VII}_{6}$ \\
\hline PsS23 & & & & & G7 & $\mathrm{VII}_{6}$ \\
\hline PsWs173 & HM627621 & $\begin{array}{l}\text { P. putida / } \\
\text { P. plecoglossicida }\end{array}$ & JN711467 & Pseudomonas sp. & G8 & $\mathrm{V}_{6}$ \\
\hline PsS28 & HM627596 & $\begin{array}{l}\text { P. putida / } \\
\text { P. plecoglossicida }\end{array}$ & JN590028 & P. oleovorans & G9 & $\mathrm{IV}_{6}$ \\
\hline PsS79 & HM627629 & $\begin{array}{l}\text { P. putida / } \\
\text { P. plecoglossicida }\end{array}$ & JN695065 & P. oleovorans & G9 & $\mathrm{IV}_{10}$ \\
\hline PsS48 & HM627598 & $\begin{array}{l}\text { P. putida / } \\
\text { P. plecoglossicida }\end{array}$ & JN590031 & P. oleovorans & G9 & $\mathrm{IV}_{9}$ \\
\hline PsS102 & & & & & G9 & $\mathrm{VII}_{2}$ \\
\hline PsTp156 & HM627607 & $\begin{array}{l}\text { P. putida / } \\
\text { P. plecoglossicida }\end{array}$ & JN590040 & P. oleovorans & G9 & $\mathrm{IV}_{3}$ \\
\hline PsWs158 & & & & & G9 & $\mathrm{IV}_{4}$ \\
\hline PsS15 & HM627595 & $\begin{array}{l}\text { P. putida / } \\
\text { P. plecoglossicida }\end{array}$ & JN634562 & P. oleovorans & G9 & $\mathrm{IV}_{1}$ \\
\hline PsC54 & HM627597 & $\begin{array}{l}\text { P. putida / } \\
\text { P. plecoglossicida }\end{array}$ & JN603372 & P. oleovorans & G10 & $\mathrm{IV}_{7}$ \\
\hline PsS4 & HM627612 & Pseudomonas sp. & JN590027 & P. plecoglossicida & G9 & $\| I_{8}$ \\
\hline Isolates & $\begin{array}{l}\text { 16S rDNA } \\
\text { accession } \\
\text { number }\end{array}$ & $\begin{array}{llr}\text { Closest } & \text { 16S } & \text { rDNA } \\
\text { sequence } & \text { with } & \text { type } \\
\text { strain } & & \end{array}$ & $\begin{array}{l}\text { rpoB } \\
\text { accession } \\
\text { number }\end{array}$ & $\begin{array}{l}\text { Closest rpoB } \\
\text { sequence with type } \\
\text { strain }\end{array}$ & $\begin{array}{l}\text { ITS } \\
\text { profile }\end{array}$ & $\begin{array}{l}\text { BOX } \\
\text { profile }\end{array}$ \\
\hline PsS46 & HM627613 & $\begin{array}{l}\text { P. putida / } \\
\text { P. plecoglossicida }\end{array}$ & JN590030 & P. oleovorans & G6 & $\mathrm{IV}_{1}$ \\
\hline PsS31 & HM627624 & $\begin{array}{l}\text { P. putida / } \\
\text { P. plecoglossicida }\end{array}$ & JN590039 & P. oleovorans & G9 & $\mathrm{VII}_{1}$ \\
\hline PsS67 & HM627602 & $\begin{array}{l}\text { P. putida / } \\
\text { P. plecoglossicida }\end{array}$ & JN695063 & P. oleovorans & G9 & $\mathrm{VII}_{3}$ \\
\hline PsS18 & & & & & G9 & $\mathrm{VII}_{3}$ \\
\hline PsWs147 & HM627623 & $\begin{array}{l}\text { P. putida / } \\
\text { P. plecoglossicida }\end{array}$ & JN620414 & P. oleovorans & G9 & $V_{5}$ \\
\hline PsS75 & HM627609 & $\begin{array}{l}\text { P. putida / } \\
\text { P. plecoglossicida }\end{array}$ & JN695064 & P. oleovorans & G9 & $V_{3}$ \\
\hline PsS83 & HM627581 & $\begin{array}{l}\text { P. putida / } \\
\text { P. plecoglossicida }\end{array}$ & JN590032 & P. corrugata & G7 & $\mathrm{Vl}_{1}$ \\
\hline PsWt146 & HM627604 & $\begin{array}{l}\text { P. putida / } \\
\text { P. plecoglossicida }\end{array}$ & JN590038 & P. monteilii & G7 & $V_{4}$ \\
\hline PsTp171 & HM627603 & P. mosselii & JN590041 & P. mosselii & G7 & $\mathrm{V}_{1}$ \\
\hline PsWw118 & HM627606 & $\begin{array}{l}\text { P. otitidis / } \\
\text { P. guezennei }\end{array}$ & JN590035 & P. otitidis & G1 & $\mathrm{VI}_{3}$ \\
\hline
\end{tabular}



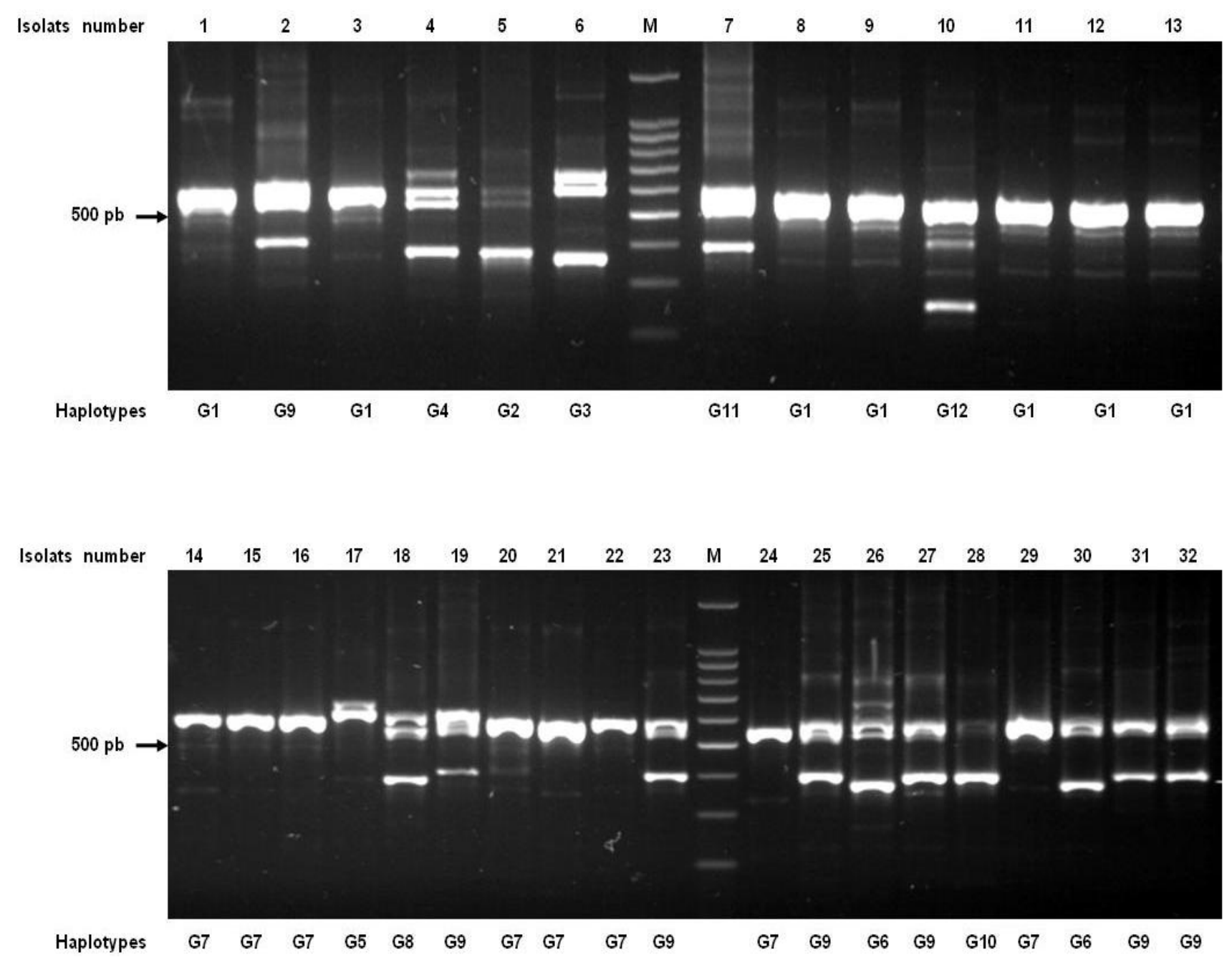

Figure 1. ITS profiles of Pseudomonas fluorescent isolates. M: 100 pb molecular size marker. Lanes 1- PsWw.118 (G1), 2PsS.31 (G9), 3- PsWw.121 (G1), 4- PsTp.153 (G4), 5- PsTp.139 (G2), 6- PsWs.140 (G3), 7- PsS.2 (G11), 8- PsS.3 (G1), 9PsS.89 (G1), 10- PsC.132 (G12), 11- PsTp.179 (G1), 12- PsWw.127 (G1), 13-PsS.150 (G1), 14- PsS.73 (G7), $15-P s S .26$ (G7), 16- PsS.93 (G7), 17- PsWw.128 (G5), 18- PsWs173 (G8), 19- PsWs.147 (G9), 20- PsTp.171 (G7), 21- PsWt.146 (G7), 22PsS.83 (G7), 23- PsS.75 (G9), 24- PsC.10 (G7), 25- PsS.4 (G9), 26- PsS.11 (G6), 27- PsS.15 (G9), 28- PsC.54 (G10), $29-$ PsS.71 (G7), 30- PsS.46 (G6), 31- PsS.79 (G9) and 32- PsS.28 (G9).

PsWw121) belong to $P$. aeruginosa, one isolate (PsWw118) belongs to P. otitidis / P. guezennei, and one isolate (PsS4) belongs to Pseudomonas sp. Phylogenetic analyses of 34 representative fluorescent pseudomonad isolates based on neighbour-joining method with 1000 bootstrap sampling resulted in three major clusters (Table 1 and Figure 3). Of the 34 isolates, cluster I was formed by 22 isolates, representing the major group, cluster II was formed by 6 strains and cluster III was formed by 6 strains.

On the basis of phylogenetic analysis of rpo $B$ gene (about 1000 bp) similarity, species such as $P$. aeruginosa, $P$. putida, $P$. plecoglossicida, $P$. monteilii, $P$. mosselii, Pseudomonas syncynea, Pseudomonas corrugata, Pseudomonas oleovorans, Pseudomonas aurantiaca, Pseudomonas chlororaphis, Pseudomonas moraviensis, $P$. otitidis and Pseudomonas vancouverensis were identified. A total of four isolates (PsS3, PsC132, PsWw84 and PsS150) belong to P. aeruginosa, two isolates (PsWw118 and PsWw121) belong to $P$. otitidis, one isolate (Ps4) belongs to $P$. plecoglossicida, 11 isolates (PsS15, PsS28, PsS79, PsTp156, PsS31, PsS46, PsS48, PsC54, PsS67, PsS75 and PsWs147) belong P. oleovorans, one isolate (PsS83) belongs to $P$. corrugata, two isolates (PsWt146 and PsWs140) belong to P. monteilii, two isolates (PsS2 and PsS71) belong to P. putida / P. syncynea, one isolate (PsTp171) belongs to P. mosselii, four isolates (PsS26, PsS29, PsS73 and PsS93) belong to $P$. aurantiaca / $P$. chlororaphis, one isolate (PsS89) belongs to $P$. 


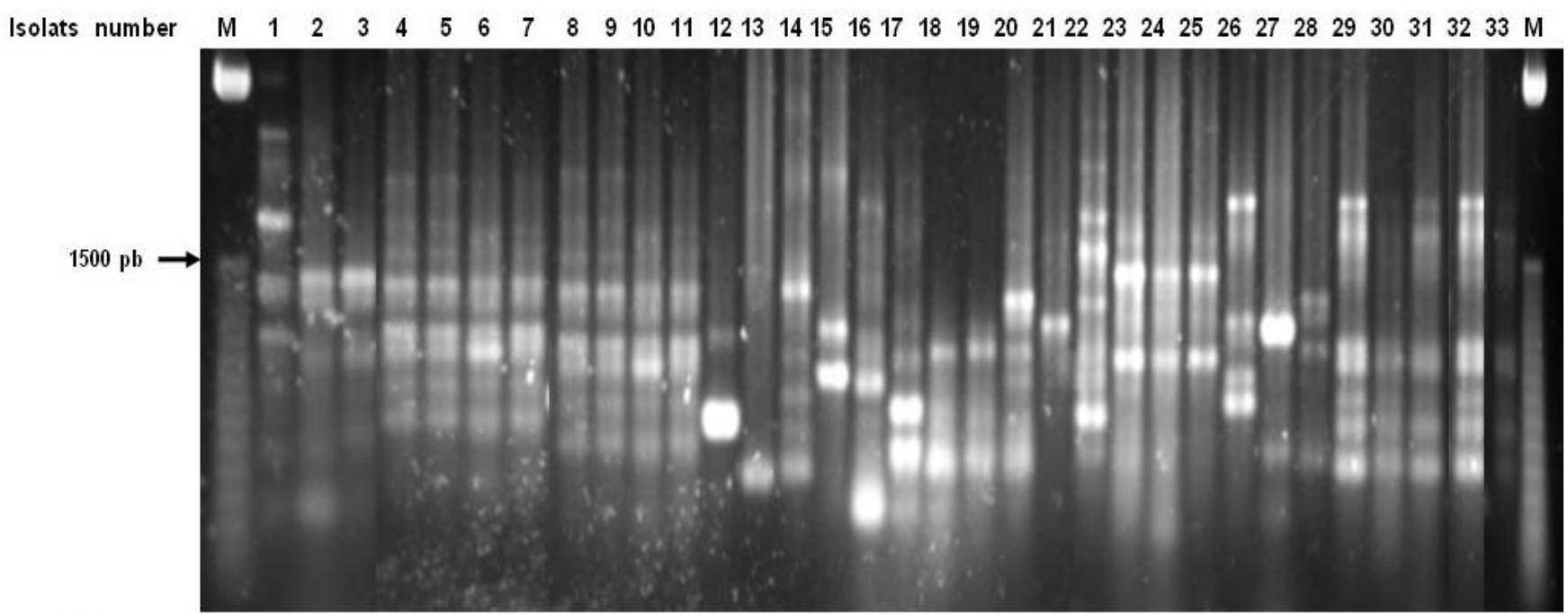

Haplotypes $\quad \mathrm{VII}_{6} \mathrm{VII}_{6} \mathrm{VII}_{6} \mathrm{VII}_{6} \mathrm{VII}_{6} \mathrm{VII}_{6} \mathrm{VII}_{6} \mathrm{VII}_{6} \mathrm{VII}_{6} \mathrm{VII}_{6} \mathrm{VII}_{6} \mathrm{VII}_{4} \mathrm{~V}_{6} \quad \mathrm{~V}_{7} \mathrm{~V}_{1} \mathrm{~V}_{4} \mathrm{VII}_{1} \mathrm{VII}_{3} \mathrm{VII}_{3} \mathrm{VII}_{2} \mathrm{VI}_{3} \mathrm{VII}_{3} \mathrm{VI}_{2} \mathrm{VI}_{2} \mathrm{VI}_{2} \mathrm{VI}_{1} \mathrm{~V}_{3} \mathrm{~V}_{2} \mathrm{IV}_{13} \mathrm{IV}_{13} \mathrm{IV}_{13} \mathrm{IV}_{13} \mathrm{IV}_{13}$

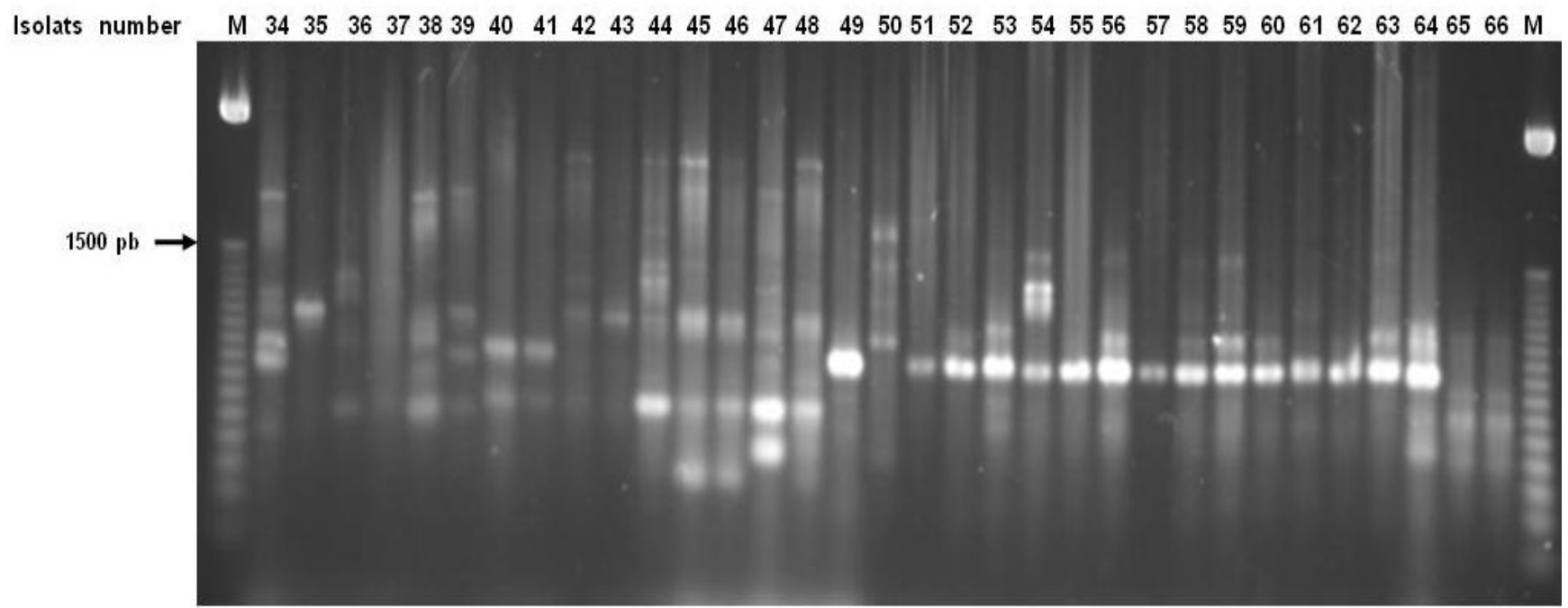

Haplotypes

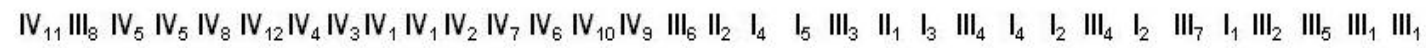

Figure 2. BOX profiles of Pseudomonas fluorescent isolates. M: 50 pb molecular size marker. Lanes 1- PsS.73 (VII6), $2-$ PsS.26 $\left(\mathrm{VII}_{6}\right)$, 3- PsS.60 ( $\left.\mathrm{VII}_{6}\right)$, 4- PsS.49 (VII $)$, 5- PsS.23 (VII $)$, 6- PsS.90 (VII $)$, 7- PsS.91 (VII $)$, 8- PsS.93 (VII $)$, 9- PsS.29 (VII $)$, $10-$

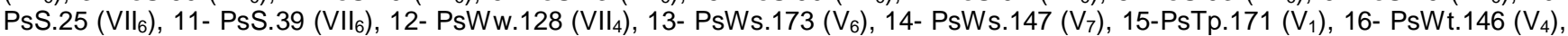
17-PsS.31(VII 1 ), 18- PsS.18 $\left(\mathrm{VII}_{3}\right)$, 19- PsS.67 $\left(\mathrm{VII}_{3}\right)$, 20- PsS.102 $\left(\mathrm{VII}_{2}\right)$, 21- PsWw.118 $\left(\mathrm{VI}_{3}\right)$, 22- PsS.89 $\left(\mathrm{VII}_{5}\right)$, $23-\mathrm{PsTp} .142(\mathrm{VI})$,

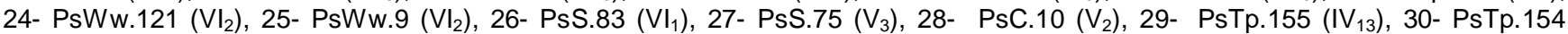

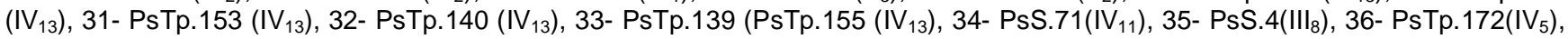

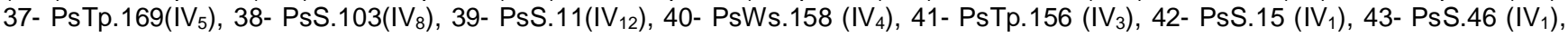
44- PsWw.124 (IV $)$, 45- PsC.54 (IV $)$, 46- PsS.28 (IV $)$, 47- PsS.79 (IV 10$)$, 48- PsS.48 (IV 9 ), 49- PsWw.84 (III $)$, 50- PsTp.160(II $)$,

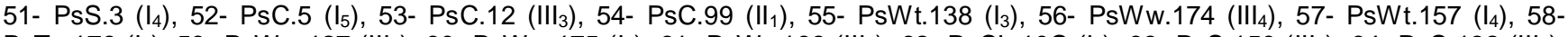
PsTp.179 $\left(\mathrm{I}_{2}\right), 59-\mathrm{PsWw} .127\left(\mathrm{III}_{4}\right), 60-\mathrm{PsWw} .175\left(\mathrm{I}_{2}\right), 61-\mathrm{PsWw168}\left(\mathrm{III}_{7}\right), 62-\mathrm{PsCL} .10 \mathrm{C}\left(\mathrm{I}_{1}\right), 63-\mathrm{PsS} .150\left(\mathrm{II}_{2}\right), 64-\mathrm{PsC} .132\left(\mathrm{III}_{5}\right)$, 65- PsS.2 (III $)_{1}$ and 66- PsCL.HMC1 (III $\left.)_{1}\right)$.

moraviensis, one isolate (PsWw128) belongs to $P$. vancouverensis and four isolates (PsC10, PsS11, PsWs173 and Ps139) belong to Pseudomonas sp. Phylogenetic analyses of 34 representative fluorescent pseudomonad isolates based on $\mathrm{NJ}$ method with 1000 bootstrap sampling resulted into three six clusters (Table 1 and Figure 4). Of the 34 isolates, cluster I contained 20 isolates, representing the major group, cluster II contained six strains, cluster III contained one strain, cluster IV contained four strains, cluster V contained two 


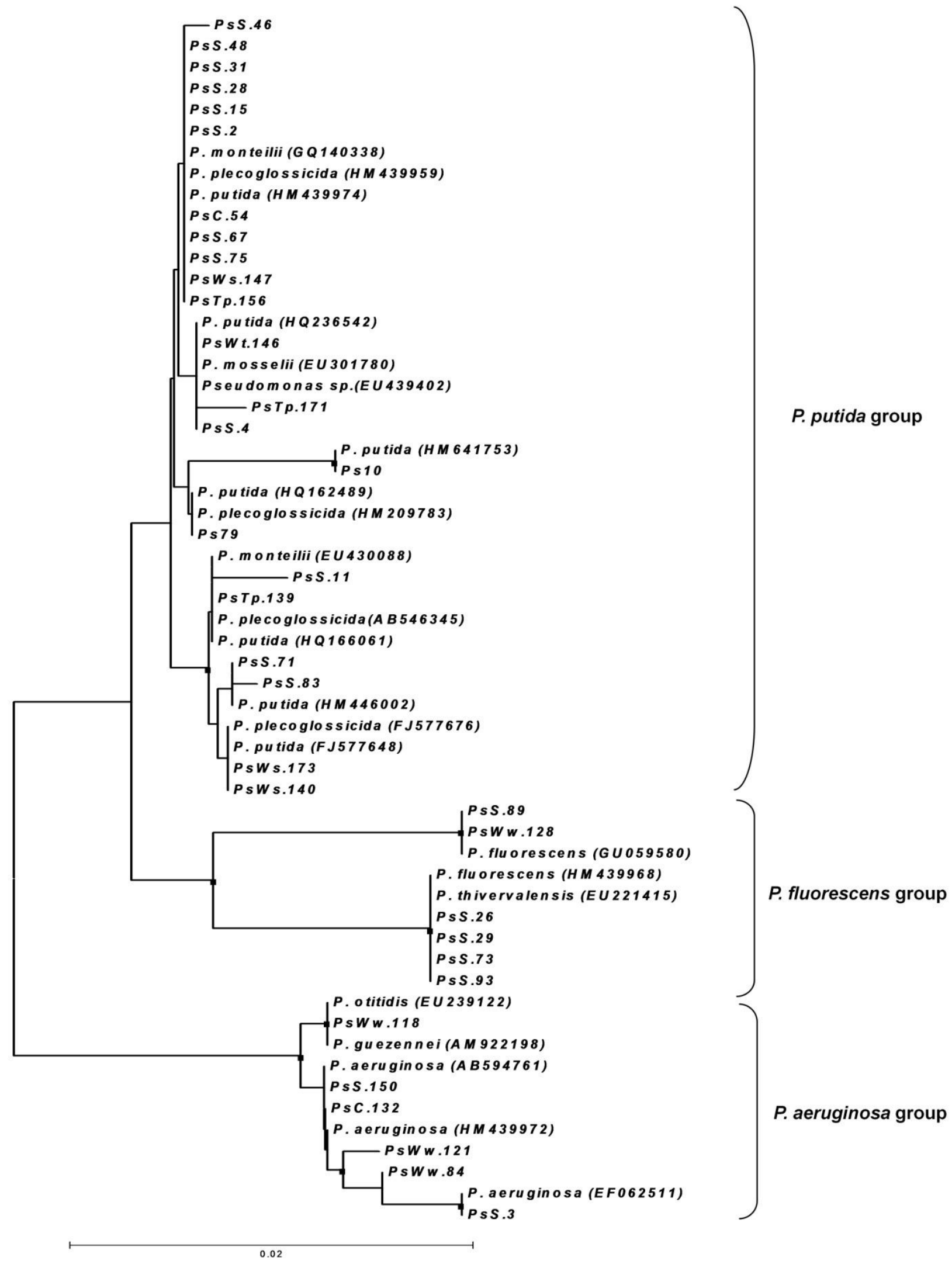

Figure 3. Neighbor-joining phylogenetic tree of 16S rRNA sequences of 34 isolates and their closest phylogenetic relatives. Sequences of the compared strains were obtained from databases, and the accession numbers are in parenthesis. The tree topology was constructed using ClustalX (1.81). 

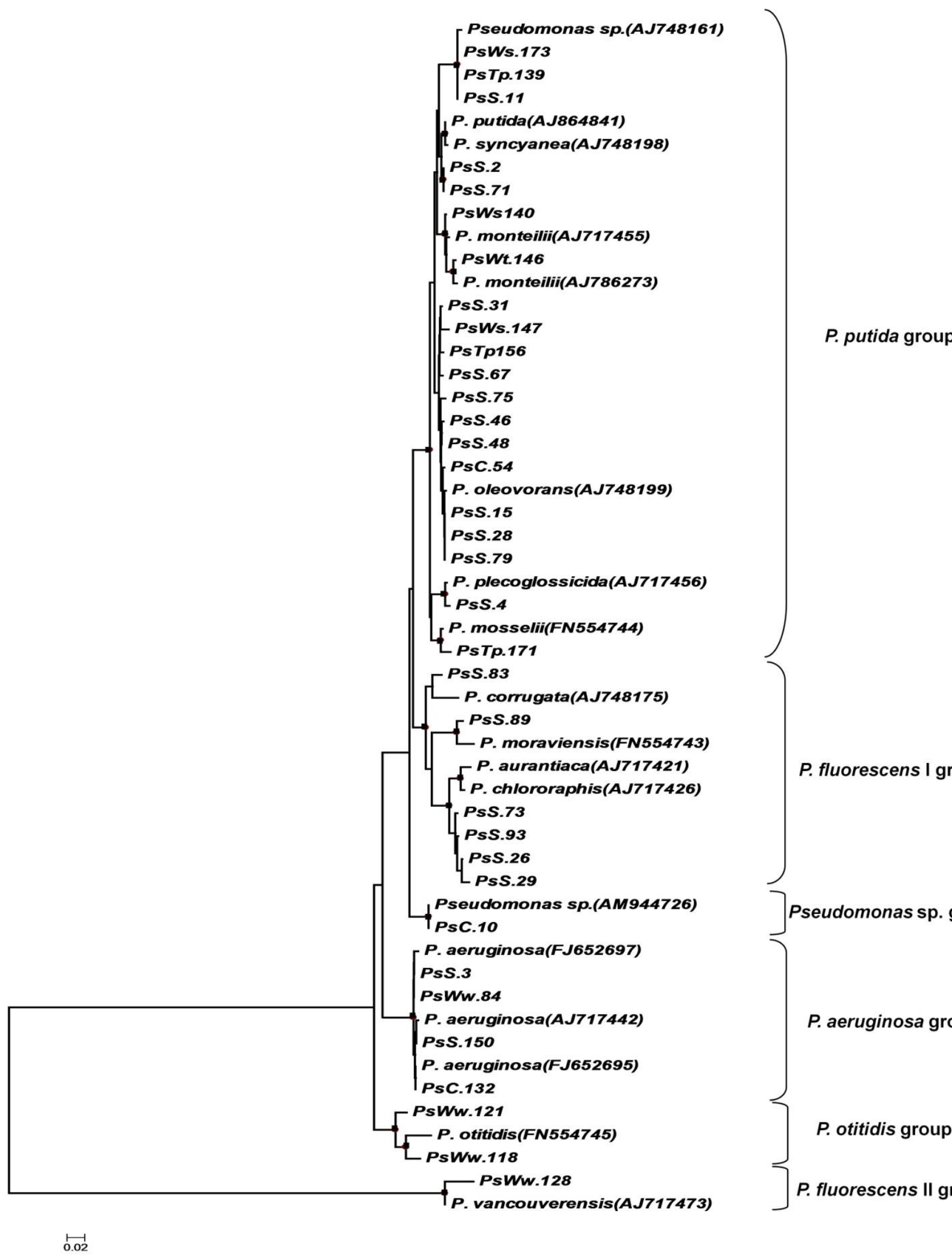

P. fluorescens I group

Pseudomonas sp. group

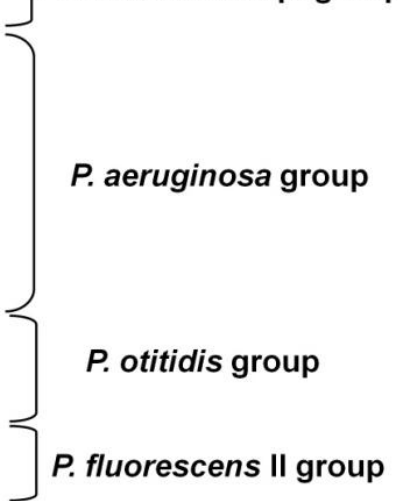

Figure 4. Neighbor-joining phylogenetic tree of $R p o B$ sequences of 34 isolates and their closest phylogenetic relatives. Sequences of the compared strains were obtained from databases, and the accession numbers are in parenthesis. The tree topology was constructed using ClustalX (1.81). 
strains and cluster VI contained one strain.

\section{DISCUSSION}

The involvement of some fluorescent Pseudomonas species in plant diseases and some others as powerful plant growth-promoting rhizobacteria, together with the demonstration of pyoverdines as biocontrol agents implicated in such processes (Meyer, 2000), shows that an improved classification must be developed.

In this investigation, BOX-PCR, using a single primer, showed a higher discriminatory power and polymorphism than ITS-PCR. Jensen et al. (1993) reported that ITS polymorphisms may form the PCR based identification of many bacterial species, as well as differentiating between certain bacteria at subspecies level. Our results differed from that in this findings, in that, the ITS-PCR typing method did not seem to be a very logical division of species when compared with the other methods. For example, PsWw.118 (identified as $P$. otitidis) and PsS.150 (identified as $P$. aeruginosa) are grouped into the same haplotype (G1). This was largely due to the limited number of bands in each group, which did not provide sufficient characters for confident differentiation of all genomovars (Dawson et al., 2002). In order to increase the number of comparable characters, other authors have found that ITS-PCR typing can be improved by the digestion of the PCR products with the restriction endonucleases (Natalini and Scortichini, 2007; Scarpellini et al., 2004).

In recent study of Popavath et al. (2008), the BOX-PCR genomic fingerprints produced $62 \mathrm{BOX}$ profiles within a total of 95 isolates of fluorescent pseudomonads isolated from banana root-adhering soil. In fact BOX-PCR genomic fingerprint was able to differentiate strains very closely related. This method allowed the recognition of 14 different profiles among the $P$. aeruginosa isolates, which reflected the high degree of interspecies diversity (popavath et al., 2008). Furthermore, a common band was observed (400 bp) in all $P$. aeruginosa isolates patterns which could allow the recognition of this specie. BOX-PCR may be better as it is targeted to a specific region of the entire genome, including the variable regions that do not undergo a large pressure selection, while ribosomal operons are highly conserved (Versalovic et al., 1991). Another study conducted by Shanmugam et al. (2008) reinstated the importance of whole-cell protein analysis in assessing the diversity of pseudomonads. Olive and Bean (1999) showed that BOX-PCR have a better strain differentiation power, easier to perform, quicker and cheaper than ribosomal intergenic spacer analysis (RISA), restriction fragment length polymorphism (RFLP) and other techniques.

Based on phylogenetic characterization, only 9 branches in the 16S rRNA tree are supported by bootstrap values above $70 \%$ while the rpoB tree contains much more branches (17 branches). Furthermore, comparison of the $16 \mathrm{~S}$ rRNA and $r p o B$ phylogenetic trees led us to observe generally longer branches in the rpoB tree than in the 16S rRNA tree (while considering the scale at 0.02). In addition, the sequencing of the rpoB gene has identified 13 different species and sub-species while the 16 rRNA gene sequencing differentiated only 9 species. Obviously, higher phylogenetic resolution was obtained with rpoB sequences. This result is in agreement with the findings of Ait Tayeb et al. (2005) that showed approximately three-times-higher taxonomic resolution of the $r p o B$ tree than that of $16 \mathrm{~S}$ rRNA tree. However, functional genes such as $r p o B$ could be used to target a more precise phylogeny and for a higher taxonomic resolution, because of its essential role in cellular metabolism (Ait Tayeb et al., 2005).

\section{Conclusions}

Results of this investigation have demonstrated that BOX-PCR show a good discriminative ability with reproducibility, stability, low cost and can be used as a rapid method of comparing fairly homogenous pseudomonads strains for genetic diversity. In addition, the $r p o B$ sequencing seems to be most suitable for identification of Pseudomonas strains at species level. The three-times-higher taxonomic resolution will facilitate assignation of a strain to a species. The $r p o B$ sequencing could significantly extend Pseudomonas phylogeny to provide better picture of Pseudomonas diversity.

\section{REFERENCES}

Adékambi T, Drancourt M, Raoult D (2009). The rpoB gene as a tool for clinical microbiologists. Trends Microbiol. 17: 37-45.

Ait Tayeb L, Ageron E, Grimont F, Grimont PAD (2005). Molecular phylogeny of the genus Pseudomonas based on rpoB sequences and application for the identification of isolates. Res. Microbiol. 156: 763-773.

Bennasar A, Mulet M, Lalucat J, Garcia-Valdés E (2010). PseudoMLSA: A database for multigenic sequence analysis of Pseudomonas species. BMC Microbiol. 10: 118.

Brusetti L, Malkhazova I, Gtari M, Tamagnini I, Borin S, Merabishvili M, Chanishvili N, Mora D, Cappitelli F, Daffonchio D (2008). Fluorescent-BOX-PCR for resolving bacterial genetic diversity, endemism and biogeography. BMC Microbiol. 8: 220.

Bultreys A, Gheysen I, Wathelet B, Maraite H, de Hoffmann E (2003). High-Performance Liquid Chromatography Analyses of Pyoverdin Siderophores Differentiate among Phytopathogenic Fluorescent Pseudomonas Species. Appl. Environ. Microbiol. 69: 1143-1153.

Chen WP, Kuo TT (1993). A simple and rapid method for preparation of gram-negative bacterial genomic DNA. Nucl. Acids Res. 21: 2260.

Costa R, Gomes NCM, Peixoto RS, Rumjanek N, Berg G, MendonçaHagler LCS, Smalla K (2006). Diversity and antagonistic potential of Pseudomonas spp. Associated to the rhizosphere of maize grown in a subtropical organic farm. Soil Biol. Biochem. 38: 2434-2447.

Dawson SL, John C Fry JC, Brian N Dancer BN (2002). A comparative evaluation of five typing techniques for determining the diversity of fluorescent pseudomonads. J. Microbiol. Meth. 50: 9-22.

Dong YH, Zhang LH (2005). Quorum sensing and quorum-quenching enzymes. J. Microbiol. 43: 101-109.

Fuchs R, Schäfer M, Geoffroy V, Meyer JM (2001). Siderotyping-a powerful tool for the characterization of pyoverdines. Curr. Top. Med. 
Chem. 1:31-35.

Hasegawa M, Hashimoto T (1993). Ribosomal RNA trees misleading? Nature 361: 23.

Hunter PR, Gaston MA (1988). Numerical index of the discriminatory ability of typing systems: An application of simpson's index of diversity. J. Clin. Microbiol. 26: 2465-2466.

Jensen MA, Webster JA, Straus N (1993). Rapid identification of bacteria on the basis of the polymerase chain reaction-amplified ribosomal DNA spacer polymorphisms. Appl. Environ. Microbiol. 59: 945-952.

King EO, Ward M K, Raney DE (1954). Two simple media for the demonstration of pyocyanin and fluorescein. J. Lab. Clin. Med. 44: 301-307.

Lane DJ, Pace B, Olsen GJ, Stahl DA, Sogin ML, Pace NR (1985). Rapid determination of $16 \mathrm{~S}$ ribosomal RNA sequences for phylogenetic analyses. Proc. Natl. Acad. Sci. USA. 82: 6955-6959.

Latour X, Delorme S, Mirleau P, Lemanceau P (2003). Identification of traits implicated in the rhizosphere competence of fluorescent Pseudomonads: Description of a strategy based on population and model strain studies. Agronomie 23: 397-405.

Louws FJ, Fulbright DW, Stephens CT, Bruijn FJ (1994). Specific genomic fingerprints of phytopathogenic Xanthomonas and Pseudomonas pathovars and strains generated with repetitive sequences and PCR. Appl. Environ. Microbiol. 60: 2286-2295.

Louws FJ, Bell J, Medina-Mora CM, Smart CD, Opgenorth D, Ishimaru CA, Hausbeck MK, de Bruijn FJ, Fulbright DW (1998). Rep-PCRmediated genomic fingerprinting: $A$ rapid and effective method to identify Clavibacter michiganensis. Phytopathology 88: 862-868.

Marques ASA, Corbiere R, Gardan L, Tourte C, Manceau C, Taylor JD, Samson R (2000). Multiphasic approach for the identification of the different classification levels of Pseudomonas savastanoi pv. phaseolicola. Eur. J. Plant Pathol. 106: 715-734.

Massart S, Jijakli HM (2007). Use of molecular techniques to elucidate the mechanisms of action of fungal biocontrol agents: A review. J. Microbiol. Meth. 69: 229-241.

Meyer JM (2000). Pyoverdines: Pigments, siderophores and potential taxonomic markers of fluorescent Pseudomonas species. Arch. Microbiol. 174: 135-142.

Meyer JM, Geoffroy V, Baida N, Gardan L, Izard D, Lemanceau P, Achouak W, Palleroni NJ (2002). Siderophore Typing, a Powerful Tool for the Identification of Fluorescent and Nonfluorescent Pseudomonads. Appl. Environ. Microbiol. 68: 2745-2753.

Migula W (1894). Über ein neues System der Bakterien. Arb. Bakteriol. Inst. Karlsruhe 1: 235-238.

Moore ERB, Mau M, Arnscheidt A, Böttger EC, Hutson RA, Collins MD, Van De Peer Y, De Wachter R, Timmis KN (1996). The determination and comparison of the 16S rRNA gene sequences of species of the genus Pseudomonas (sensu strictu) and estimation of the natural intrageneric relationships. Syst. Appl. Microbiol. 19: 478-492.
Moreira L, Agostino P, Morais PV, da Costa MS (1994). Survival of allochthonous bacteria in still mineral water bottled in polyvinyl chloride (PVC) and glass. J. Appl. Bacteriol. 77: 334-339.

Munsch P, Geoffroy V, Alatossava T, Meyer JM (2000). Application of siderotyping for characterization of Pseudomonas tolaasii and "Pseudomonas reactans" isolates associated with brown blotch disease of cultivated mushrooms. Appl. Environ. Microbiol. 66: 48344841.

Natalini E, Scortichini M (2007). Variability of the 16S-23S rRNA gene internal transcribed spacer in Pseudomonas avellanae strains. FEMS Microbiol. Lett. 271: 274-280.

Olive DM, Bean P (1999). Principles and applications of methods for DNA-based typing of microbial organisms. J. Clin. Microbiol. 37: 1661-1669.

Ouzari H, Khsairi A, Raddadi N, Jaoua L, Hassen A, Zarrouk M, Daffonchio D, Boudabous A (2008). Diversity of auxin- producing bacteria associated to Pseudomonas savastanoi-induced olive knots. J. Basic Microbiol. 48: 1-8.

Popavath RN, Nirakar S, Devrishi G, Niraikulam A, Natarajan S (2008). Genetic and Functional Diversity among Fluorescent Pseudomonads Isolated from the Rhizosphere of Banana. Microbiol. Ecol. 56: 492 504.

Ravi Charan A, Prathap Reddy V, Narayana Reddy P, Sokka Reddy S, Sivaramakrishnan $S$ (2011). Assessment of genetic diversity in Pseudomonas fluorescens using PCR-based methods. Biorem. Biodiv. Bioavail. 5: 10-16.

Scarpellini M, Franzetti L, Galli A (2004). Development of PCR assay to identify Pseudomonas fluorescens and its biotype. FEMS Microbiol. Lett. 236: 257-260.

Shanmugam A, Mahalakshmi TS, Vino AB (2008). Antimicrobial activity of polysaccharide isolated from the cuttlebone of Sepia aculeata (Orbingy, 1848) and Sepia brevimana (Steenstrup, 1875): An approach to selected antimicrobial activity for human pathogenic microorganisms. J. Fish. Aquat. Sci. 3: 268-274.

Tacao M, Alves A, Saavedra MJ, Correia A (2005). BOX-PCR is an adequate tool for typing Aeromonas sp. Antoine Van Leeuwenhoek. 88: 173-179.

Versalovic J, Koeuth Y, Lupski JR (1991). Polymerase chain reaction with consensus sequences primers is a powerful method for typing bacteria. Nucl. Acids Res. 19: 6823-6831.

Wang L, Jayarao BM (2001). Phenotypic and genotypic characterization of Pseudomonas fluorescens isolated from bulk tank milk. J. Dairy Sci. 84: 1421-1429.

Widmer F, Seidler RJ, Gillevet PM, Watrud LS, Di Giovanni D (1998). A highly selective PCR protocol for detecting 16S rRNA genes of the genus Pseudomonas (Sensu stricto) in environmental samples. Appl. Environ. Microbiol. 64: 2545-2553. 